\title{
Salmonella Typhi Infections and Effect of Fluroquinolones and Third Generation Cephalosporins in Clinical Outcome
}

\author{
Singh UK ${ }^{1}$, Neopane $\mathrm{AK}^{2}$, Thapa $\mathrm{M}^{3}$, Aryal ${ }^{4}$, Agrawal $\mathrm{K}^{5}$
}

${ }^{1}$ Dr. Umesh Kumar Singh, MBBS. MD, ${ }^{2}$ Dr. Arun Kumar Neopane. MBBS. MD. FAAP. Associate Professor (NAMS). ${ }^{3}$ Dr. Moon Thapa, MBBS. MD, ${ }^{4}$ Dr. Nirjala Aryal, MBBS. MD. ${ }^{5}$ Dr. Keshav Agrawal, MBBS. MD. All from the Department of Paediatrics, Shree Birendra Hospital, Chhauni, Kathmandu, Nepal.

Address for correspondence: Dr. Umesh Kumar Singh, E-mail: kumar3130@yahoo.co.in

\begin{abstract}
Background: Enteric fever is a common public health problem in Nepal. The emerging fluoroquinolone resistance to Salmonella typhi is a major concern in every hospital and is a public health problem these days. Continuous antibiotic susceptibility patterns surveillance and standard treatment policies need to be established to control MDR typhoid. Objective: To detect the increasing pattern of fluoroquinolone resistant Salmonella typhi and to correlate its clinical response to third generation cephalosporins. Materials and Method: This is a cross- sectional prospective study conducted in the pediatric ward of Birendra Hospital, Kathmandu, from September 2009 to August 2010. Forty seven children aged between 3-14 years with the diagnosis of suspected, probable and confirmed cases of Enteric fever were enrolled in the study. Data was collected and statistical analysis was done using SPSS program. Result: Culture positive enteric fever was found in 21 cases (44.68\%) and positive Widal test in 18 (38.29\%). Among the culture positive cases, antibiotic sensitivity was highest for Ofloxacin (95.23\%), followed by third generation Cephalosporins (Ceftriaxone, Cefixime) (90.47\%). A significant number of isolates were resistant to Nalidixic acid $(71.42 \%)$. All cases were successfully treated with parenteral antibiotics followed by oral third generation cephalosporins without any complications and/or mortality. Conclusion: There is an increasing trend to fluoroquinolone resistant Salmonella typhi. Third generation cephalosporin can be the appropriate antibiotic for treatment.of clinically suspected cases of Enteric fever and to minimize the risk of increasing emergence of MDR enteric fever including Nalidixic acid resistant Typhoid.(NARST).
\end{abstract}

Key words: Cephalosporin, drug resistance, Enteric fever, Fluoroquinolone, Salmonella typhi.

\section{Introduction}

Enteric fever (more commonly termed typhoid fever) remains endemic in many developing countries. Typhoid fever is caused by Salmonella enteric serovar Typhi (S.typhi) which is a gram negative, flagellate, non-sporulating, facultative anaerobic bacilli that ferment glucose, reduce nitrate to nitrite and synthesize peritrichous flagella when motile. Salmonella is a genus in the family Enterobacteriaceae that has more than 2300 serotypes, based on the presence of three main antigens: somatic $\mathrm{O}$ antigen (lipopolysaccharide cell wall component), surface virulent (vi) antigen (S.Typhi and S.Paratyphi $\mathrm{C}$ only), and flagellar $\mathrm{H}$ antigen ${ }^{1}$. A very similar but often less severe disease is caused by $S$. Paratyphi A and rarely by S.Paratyphi B and S.Paratyphi
C. The ratio of disease caused by S.typhi to that caused by others is about 10 to 1 in most countries. ${ }^{2}$ Enteric fever may occur at any age but is more prevalent in children and young adults. The peak incidence is seen in children between 5-15 years of age. According to hospital-based data and limited information from field studies that used passive surveillance for case detection, typhoid fever is infrequent under 3 years of age ${ }^{3-6}$. It has become rare in industrialized countries, yet remains a major cause of enteric disease in children in developing countries ${ }^{7}$, resulting in an estimated incidence of 50 cases per 100,000 persons per year, predominantly in young school-aged children ${ }^{8}$. Cases occur throughout the year but increase during summers, the peak months being July to September. It particularly occurs soon after 
community parties and festivals where large numbers of people gather. ${ }^{9}$ Infection with salmonella serovar Typhi causes an estimated 20 million cases of typhoid fever and 200,000 deaths annually worldwide. Altogether the risk of typhoid fever may be reduced with attention to water quality, food hygiene and vaccination; effective treatment of S. typhi infection is needed when these measures fail.

Over the last 20 years, emergence of S.Typhi strains resistant to antimicrobial agents has complicated treatment of infected patients ${ }^{10}$. Multiple drug resistant(MDR) S. Typhi denotes those strains of typhoid fever which have resistant to all the three first line antibiotics (Chloramphenicol, Ampicillin and Cotrimoxazole $)^{9}$. Although $3^{\text {rd }}$ generation Cephalosporin still has good result against S.Typhi, increasing prevalence of multi drug resistant (MDR) S.Typhi attributes a major public health problem in underdeveloped countries like ours, where mainstay of therapy are drugs like Cotrimoxazole, Amoxycillin and Chloramphenicol. Besides these first line antibiotics, widespread use of fluoroquinolones during outbreaks of Nalidixic acid-resistant typhoid showing reduced susceptibility to fluroquinolones, may potentiate the already existing MDR typhoid further worse.

\section{Materials and methods}

This is a cross- sectional prospective study conducted in the paediatric ward of Birendra Hospital (Chhauni).

Study population: Febrile children, aged between 5 to 14 years, having any of the clinical features of suspected, probable or confirmed enteric fever, admitted to Shree Birendra Hospital (Chhauni) from September 2009 to August 2010, were included in the study.. Children belonging to army personnel were included in this study as this hospital is designated for serving them. None of the study population had been vaccinated with Typhoid vaccine. The children with the presence of symptoms of enteric fever were admitted to $\mathrm{n}$ the paediatric ward. Investigations were sent to the hospital laboratory. Consent was taken from parents/ caretakers.

\section{Enrollment}

The clinical case definition (according to the Indian Academy of Pediatrics guidelines) of suspected enteric fever was a stepladder pattern of fever, headache, malaise with loss of appetite, usually with gastrointestinal symptoms, and having two or more of the following symptoms/signs: toxic look; relative bradycardia; rose spots on the trunk; splenic enlargement; or nonproductive cough $^{11,12}$. A probable case of typhoid fever was a patient with fever lasting for at least three days with a positive serodiagnosis but without $\mathrm{S}$. typhi isolation. A confirmed case was a suspected/probable case that was confirmed by positive blood culture for S. typhi after 72 hours of incubation.. For optimal result, Widal test was done after 5 to 7 days of fever by slide agglutination method and level of both $\mathrm{H}$ and $\mathrm{O}$ antibodies of 1 in 160 dilution (four fold rise) was taken as cut off value for diagnosis. ${ }^{9} \mathrm{All}$ the enrolled patients did not have any antibiotics for the fever. Pallor was considered among those patients who met the WHO criteria; diarrhoea, cough, anorexia, abdominal pain, headache and vomiting as mentioned by parents or caretakers; coated tongue, hepatomegaly and splenomegaly as noted on examination; toxic look for lethargic, ill looking appearance and febrile children with very high temperature $\left(>41^{\circ} \mathrm{C}\right)$ were enrolled in this study.

\section{Results}

Out of the total 47 children studied, 25 (53.19\%) and $19(40.42 \%)$ children were between the age of $5-10$ years and older respectively, with male to female ratio of 1.23. Thirty seven $(78.72 \%)$ cases were admitted in the month of April to August.

There was no difference seen in the common clinical features of enteric fever in the study population. Most frequent clinical symptoms and signs included fever $(95.74 \%)$, toxic look $(61.70 \%)$, splenomegaly $(59.57 \%)$, coated tongue $(57.44 \%)$, hepatomegaly (53.19\%), vomiting (46.80\%), headache $(42.55 \%)$ and abdominal pain (42.55\%). Diarrhoea (6.38\%) and pallor (4.25\%).

Twenty one blood samples (44.68\%) yielded Salmonella, of which $14(66.66 \%)$ samples were collected during the first week of fever, $5(23.80 \%)$ during the second week and remaining $2(9.52 \%)$ samples after 2 weeks of fever.Widal test was found positive in only 18 (38.29\%) cases. Positive blood culture with positive antibodies were seen among 9 (19.14\%) children.

Among the culture positive samples, Ofloxacin was found to be the most sensitive antibiotics in vitro $(95.23 \%)$ followed by Ceftriaxone and Cefixime (90.47\%),Amoxycillin (80.95\%), Chloramphenicol $(80.95 \%)$, Ciprofloxacin $(71.42 \%)$ and Cotrimoxazole $(66.66 \%)$. Although fluoroquinolones, especially Ofloxacin is significantly sensitive to salmonella isolates, Nalidixic acid remained outstandingly resistant $(71.42 \%)$ in this study, which is statistically very important. 


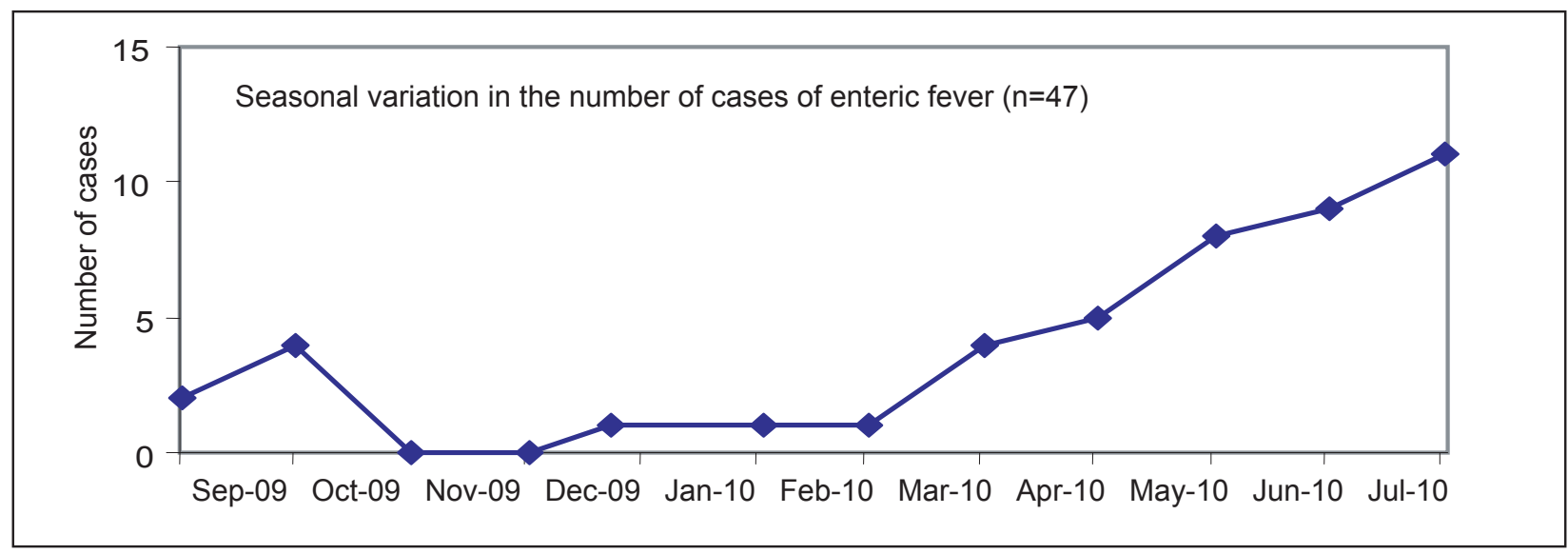

Fig 1: Seasonal variation in the number of cases of enteric fever.

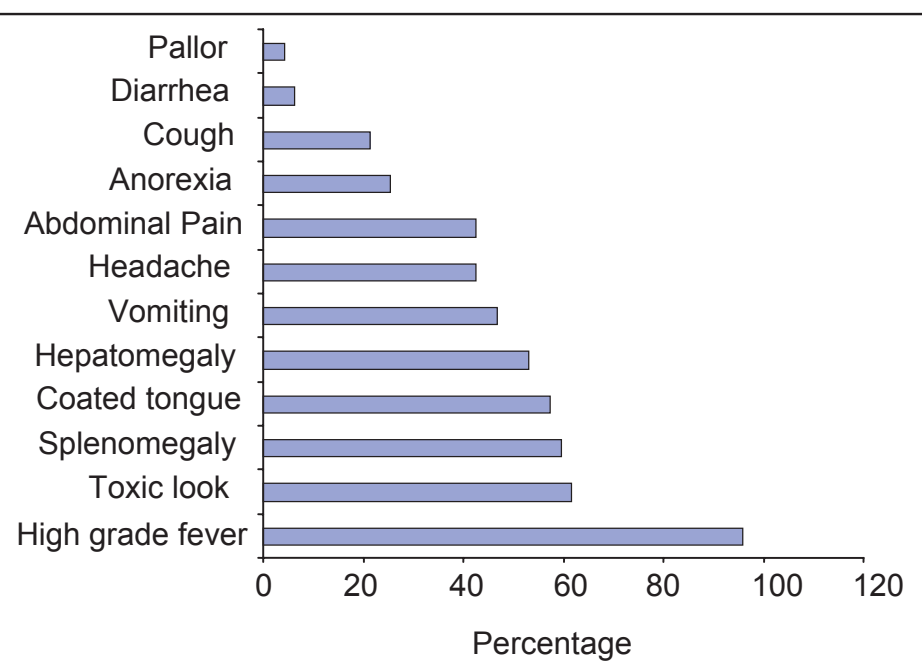

Fig 2: Pattern of Clinical features

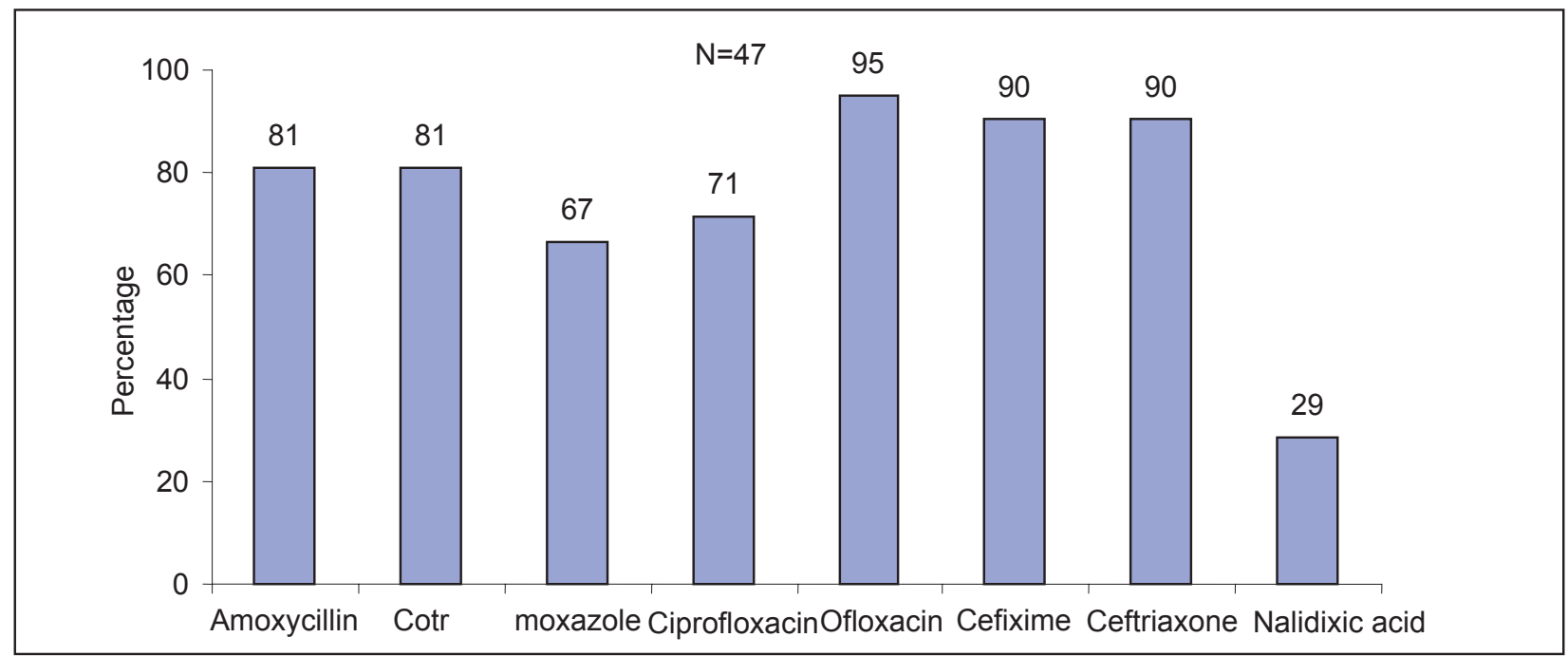

Fig 3: Susceptibility patterns of S. Typhi isolates

All the children received parenteral third generation Cephalosporin (Ceftiaxone/cefotaxime) for 7 to 10 days and were discharged without any complications. As depicted in the table below, maximum number of children had a febrile period of 3 to 5 days with mean time-to- fever defervescence of $3.68 \pm 1.1$ days (range: 1-6 days). Only 1 patient became afebrile from second day while none of the patients had fever beyond the seventh day of admission. 
Table 1: Duration of fever after starting Antibiotics

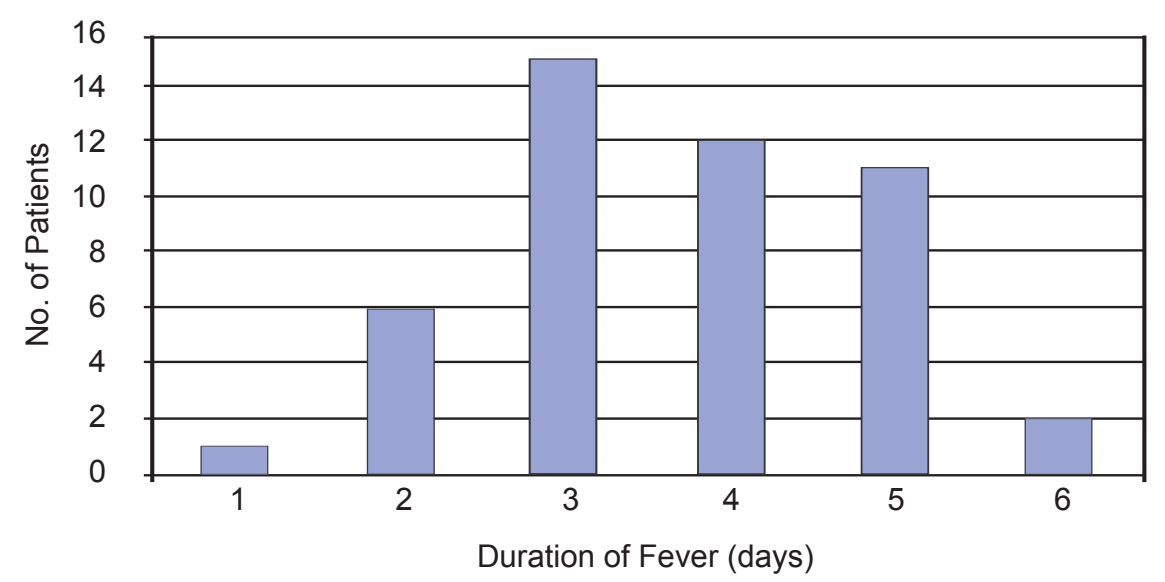

\section{Discussion}

It is known that the disease peaks around monsoon months and similar results has been shown in this study with an admission of $78.72 \%$ of the cases from the month of April to August. the study conducted in Kathmandu for 3 years demonstrated an association with the average monthly rainfall and a peak in the seasonal temperatures, with an obvious increase in cases between June and August and a maximum in July ${ }^{13}$. The study done by Sinha et al in Kalkaji, New Delhi showed that the maximum incidence (18.8 cases per 1000 person-years) occurred during the monsoon (July -October), and lower rates of 5.4 and 4.7 per 1000 person-years occurred during summer and winter seasons respectively ${ }^{14}$.

In our study, besides fever, toxic look (60.70\%), splenomegaly (59.57\%), coated tongue (57.44\%) and hepatomegaly $(53.19 \%)$ were the major physical findings. In another study, headache, relative bradycardia and myalgia were the leading clinical sign followed by coated tongue, dehydration and abdominal pain, while hepatomegaly and splenomegaly were found only in $17.5 \%$ and $12.3 \%$ respectively ${ }^{15}$. Positive blood culture in this study was $44.66 \%$; two third of which was yielded on the first week of fever. Similar studies has shown only $22 \%$ cases confirmed by blood culture ${ }^{15}$. In Another study in Ethiopia, blood culture was positive in 34 out of 54 cases with $63 \%$ positivity rate ${ }^{16}$. Although, positive blood culture is the gold standard for diagnosis of Typhoid fever, different serological tests should be strongly considered for diagnosis and early treatment; and even clinically suspected cases that meet the diagnostic criteria needs to be considered for treatment in resource poor-settings where diagnostic facilities are limited. Half of false negative widal results probably indicates suboptimal sensitivity result which might be due to negativity in early infection, prior antibiotic therapy, failure to mount an immune response by certain individuals or poor standardization of antigens used for the widal test. Literatures show that it can be negative in upto $30 \%$ of culture proven cases of typhoid fever ${ }^{9}$.

Although our report poses significant antibiotic susceptibility in vitro towards Ofloxacin, great proportionality of resistance to Nalidixic Acid has justified the ontowards effect of ever increasing public health problem of MDR typhoid. Similar studies conducted in UK during 1990s shows increasing trend of resistance to Ciprofloxacin from $2.7 \%$ in 1995 to $21 \%$ in 1998 , the majority of patients from whom such strains were isolated had recently returned from the Indian Sub-continent. ${ }^{17}$ Nine out of 63 culture positive cases studied by Sinha et al which had shown $96 \%$ sensitivity to Ciprofloxacin in vitro, did not recover after a 10-day course of the drug and had to be treated with Ceftriaxone ${ }^{14}$. Outbreaks of Nalidixic Acid resistant typhoid with isolates showing reduced susceptibility to fluoroquinolones started to occur in Vietnam ${ }^{18}$ and Tazikstan ${ }^{19}$ in the early 1990s and then spread to Pakistan and India and several other countries in Southeast $\mathrm{Asia}^{20-23}$, Kuwait ${ }^{24}$ and $\mathrm{Nepal}^{25}$; Often treatment of typhoid with Ofloxacin and Ciprofloxacin resulted in delayed clinical response and sometimes treatment failure. Ceftriaxone is found to be the effective antibiotic for hospital based treatment of typhoid fever, which also has equal susceptibility in vitro(90.47\%) along with Cefixime. This study also showed that the mean time-to-fever defervescence was $3.68 \pm 1.1$ days (range: 1-6 days). Similar results were found by Aypak $A$ et al in which treatment with Ceftriaxone showed mean time-to-fever defervescence of $3.5 \pm 1.5$ days (range : $1-8$ days) ${ }^{15}$. On virtue of fluoroquinolones resistance these days, $3^{\text {rd }}$ generation parenteral as well as oral Cephalosporins could still be the drug of choice for hospital based treatment. 


\section{Conclusion}

The increasing trend of isolation of MDR Salmonella typhi to fluoroquinolones is a major concern in the treatment of typhoid especially in resource poor settings. Injudicious use of antibiotics, over-thecounter prescriptions and sale of antibiotics, limited facilities for diagnosis, poor surveillance systems are the mainstreams responsible for the steady increase in MDR typhoid. The burden of hospital admissions can be reduced by appropriate use of third generation oral cephalosporins and determination of optimum treatment policies with continual surveillance of antibiotics susceptibility patterns and their geographical variations. Large number of cases occurring during monsoon season indicates the high risk of cross-contamination of drinking water with sewage pipelines Altogether the risk of typhoid fever may be reduced with attention to water quality, food hygiene and vaccination; effective treatment of $\mathrm{S}$. typhi infection is needed when these measures fail.

\section{Acknowledgement: None \\ Funding: None \\ Conflicting Interests: None \\ Permission from IRB: Yes}

\section{References}

1. Kanungo S, Dutta S, Sur D. Epidemiology of typhoid and paratyphoid fever in India. J Infect Developing Countries 2008;2(6): 454-60.

2. Bhutta ZA. Current concepts in the diagnosis and treatment of typhoid fever. Br Med J 2006;333:7882.

3. Sen SK, Mahakur AC. Enteric fever-a comparative study of adult and paediatric cases. Indian J Pediatr 1972;39:354-60.

4. Ferreccio C, Manterola A, Prenzel I, et al. Benign bacteremia caused by Salmonella typhi and Paratyphi in children younger than 2 years. $J$ Pediatr 1984;104:899-901.

5. Kapoor JP, Mohan M, Talwar V, et al. Typhoid fever in young children. Indian Pediatr 1985;22:811-13.

6. Mahle WT, Levine MM. Salmonella typhi infection in children younger than five years of age. Pediatr Infect Dis J 1993;12:627-31.

7. Ivanoff $B$, Levine $\mathrm{MM}$, Lambert $\mathrm{PH}$. Vaccination against typhoid fever: present status. Bull WHO 1994;72:957-91.
8. Crump JA, Luby SP, Minta ED. The global burden of typhoid fever. Bull WHO 2004;82:346-53.

9. Chitkara AJ, Adlakha N. Typhoid Fever in Children. In: Ghosh TK, Yewale V, Parthasarathy A, Shah NK editors. Pediatric Infectious Diseases (Under IAP Action Plan 2006). Mumbai: Indian Academy of Pediatrics; 2006. p 208-214.

10. Lynch MF, Blanton EM, Bulens S. Typhoid fever in United States. JAMA.2009;302(8):859- 65.

11. National Institute of Communicable Diseases Standard Case Definitions for epidemic prone diseases. CD Alert 2001;5:1-8.

12. National Institute of Communicable Diseases Investigation and Control of Water Borne Diseases. Directorate General of Health Services, 22 Shamnath Marg, New Delhi 1998;1-33.

13. Karki A, Aryal A, Basnet B, Baker S. Kathmandu, Nepal: Still an enteric fever capital of the world. J Infect Developing Countries 2008;2(6):461-65.

14. Sinha A, Sazawal S, Kumar R, et al. Typhoid fever in children aged less than 5 years. The Lancet 1999;354:734-37.

15. Aypak A, Celik AK, Aypak Cenk,Cikman O. Multidrug resistant typhoid fever outbreak in Ercek Village- Van, Eastern Antolia, Turkey: clinical profile, sensitivity patterns and response to antimicrobials. Tropical Doc 2010;40:160-62.

16. Kariuki S. Typhoid fever in sub-Saharan Africa: Challenges of diagnosis and management of infections. J Infect Developing Countries 2008;2(6):443-47.

17. Threlfall EJ, Ward LR, Skinner JA, Smith HR, Lacey S. Ciprofloxacin-resistant Salmonella typhi and treatment failure. The Lancet 1999:353:1590-91.

18. Parry CM, Hoa NTT, Diep TS, Wain J, Chinh NT, Vinh $\mathrm{H}$, Hien TT, White NJ, Farrar JJ. Value of a single-tube widal test in diagnosis of Typhoid fever in Vietnam. J Clin Microbiol 1999;37:2882-86.

19. Murdoch DA, Banatvaia N, Bone A, Shoismatulloev BI, Ward LR, Threlfall EJ. Epidemic Ciprofloxacinresistant Salmonella typhi in Tazikistan. The Lancet 1998;351:339.

20. Parry SM, PImer SR, Slader J, Humphrey T. South East Wales Infectious Disease Liaison Group: Risk factors for salmonella food poisoning in the 
domestic kitchen-a case control study. Epidemiol Infect 2002;129:277-85.

21. Pokharel BM, Koirala J, Dahal RK, Mishra SK, Khadga PK, Tuladhar NR. Multidrug-resistant and extended-spectrum beta-lactamase (ESBL)producing Salmonella enterica (serotypes Typhi and Paratyphi A) from blood isolates in Nepal: surveillance of resistance and a search for newer alternatives. Int J Infect Dis 2006;10:434-38.

22. Rahman M, Siddique AK, Shoma $S$, Rashid $H$, Salam MA, Ahmad QS, Nair GB, Breiman RF. Emergence of multidrug- resistant Salmonella enterica serotype Typhi with decreased ciprofloxacin susceptibility in Bangladesh. Epidemiol Infect 2006;134:433-38.
23. Renuka K, Sood S, Das BK, Kapil A. Highlevel ciprofloxacin resistance in Salmonella enterica serotype Typhi in India. J Med Microbiol 2005;54:999-1000.

24. Dimitrov T, Udo EE, Albaksami O, Kilani AA, Shehab DM. Ciprofloxacin treatment failure in a case of typhoid fever caused by Salmonella enterica serotype Paratyphi A with reduced susceptibility to ciprofloxacin. J Med Microbiol 2007;56:277-79.

25. Lewis H. Outbreak control team. International outbreak of Salmonella Goldcoast infection in tourists returning from Majorca, SeptemberOctober 2005: final summary. Euro Surveill. 2005; 10: E051208.3.

\section{How to cite this article?}

Singh UK, Neopane AK, Thapa M, Aryal N, Agrawal K. Salmonella Typhi Infections and Effect of Fluroquinolones and Third Generation Cephalosporins in Clinical Outcome. J Nepal Paediatr Soc 2011;31(3):216-221. 